\title{
"LA CIENCIA NO PIENSA". HEIDEGGER Y LA TAREA DE LA FILOSOFÍA COMO AUTOCONSCIENCIA
}

\section{"SCIENCE DOES NOT THINK". HEIDEGGER AND THE TASK OF PHILOSOPHY AS SELF- CONSCIOUSNESS}

\author{
Javier Castillo Vallez ${ }^{1}$ \\ Universidad de Chile, Chile
}

\begin{abstract}
Resumen: En este artículo se expone una forma de interpretar la frase de Heidegger "la ciencia no piensa" (GA 8, p. 9), de modo tal que a través de ella el autor distingue el quehacer científico y filosófico, a la vez que expone la naturaleza reflexiva o autoconsciente de esta. Con lo cual, se sostendrá que el pensamiento de Heidegger se enmarca en una cierta tradición de pensamiento socrático-kantiano que considera la naturaleza eminentemente autoconsciente de la filosofía a partir de la que se vincula con ella misma (la filosofía) y con nosotros mismos a través de ese ejercicio.
\end{abstract}

Descriptores: Pensar · Ciencia · Criterios de corrección · Autoconsciencia

\begin{abstract}
In this article a way of interpreting Heidegger's phrase "science does not think" (GA 8, p. 9) is exposed, in such a way that through it the author distinguishes scientific and philosophical work, while exposing the reflexive or self-conscious nature of this. Thus, it will be argued that Heidegger's thought is framed in a certain tradition of Socratic-Kantian thought that considers the eminently self-conscious nature of philosophy from which it is linked with itself (philosophy) and with ourselves through that exercise.
\end{abstract}

Keywords: Thinking $\cdot$ Science $\cdot$ Criteria of correctness $\cdot$ Self-consciousness

Enviado: 19/03/2021. Aceptado: 18/05/2021

\section{INTRODUCCIÓN}

Por todos es conocida la frase de Heidegger "la ciencia no piensa" (GA 8, p. 9) que ha dado lugar a muchos comentarios en sorna con la intención de remarcar la superioridad de la filosofía sobre la ciencia. Aunque no parece exacto afirmar que la ciencia "no piensa en absoluto", toda vez que el quehacer científico ha sido muy abundante y productivo tanto en la actualidad como en tiempos de Heidegger. Por lo que, el presente artículo se propone clarificar el sentido de esta controvertida afirmación. Mi hipótesis es que, con ella, Heidegger nos conduce a

\footnotetext{
${ }^{1}$ Licenciado en Filosofía, Universidad de Chile. Programa de Magíster en Filosofía. Universidad de Chile.
} 
dos asuntos cruciales, a saber, en primer lugar, entender qué caracteriza el quehacer científico, cuáles son sus límites y peculiaridades y, en segundo lugar, en qué forma se desmarca del conocimiento filosófico, cuyo modo de trabajo es completamente diferente al científico. Para ello, debemos entender en profundidad a qué llama Heidegger "pensar", distinguiéndolo de otros "tipos de pensar", no propiamente filosóficos. En último término, este artículo intenta asentar la idea de que "pensar", en rigor estricto, tiene un componente reflexivo, de manera tal que la filosofía se distinguiría de otras disciplinas en la medida en que tiene un doble objeto, a saber, un determinado objeto y ella misma, investigando dicho objeto. Este segundo orden rebasa la mera consideración del primero y aleja a la filosofía de la ciencia. Con lo cual, y contra la opinión explícita del autor, esta visión sobre la filosofía no se diferencia tanto de otros proyectos que ponen como tema central de ésta, el autoconocimiento, tales como Sócrates (Apología, 20c3-23c) o Kant (KrV, A xi-xii).

Para apoyar esta hipótesis acudiré a la obra misma de Heidegger con el fin de rastrear concepto tales como "pensamiento", "ciencia" y "meditación", a partir de textos como ¿Qué significa pensar? (2010) y Serenidad (2002). Pero, sobre todo, me interesa comparar ciertos pasajes de estas obras con otros proyectos, como los de Sócrates o Kant, con el objetivo de considerar el carácter reflexivo o autoconsciente del pensar en Heidegger.

\section{1. ¿QUÉ SIGNIFICA PENSAR?}

Si pretendemos distinguir entre "dos modos de pensar", es pertinente dilucidar qué significa pensar. Felizmente contamos con apuntes tomados de las propias lecciones del filósofo, a partir del texto ¿Qué significa pensar? (2010). La forma de proceder de Heidegger en este texto no es directa, a saber, en ninguna parte de éste intenta entregar una definición taxativa de "pensar". No obstante, sí es posible hallar ciertos indicios que nos señalan el camino para llegar a resolver tan enmarañado asunto.

\subsection{FilOSOFÍA COMO DISCIPLINA DONDE SE MANIFIESTA EL PENSAR.}

Ya en la primera lección, Heidegger nos orienta hacia una respuesta afirmando: "nos adentramos en lo que es pensar cuando pensamos nosotros mismos" (GA 8, p. 3. Énfasis mío). Con ello, el autor destaca el componente reflexivo del pensar, puesto que para adentrarnos en la cuestión del pensar es necesario que cada uno piense y medite en torno a esto. De modo que, nos encontramos en medio del siguiente problema: el modo mediante el cual debemos responder a la pregunta "qué significa pensar" es “pensando". De esta manera, no contamos con elementos externos al pensar mismo para indagar en este problema; no contamos con ninguna 
piedra de toque que nos permita obtener criterios de corrección que puedan establecer cuándo la tarea está correctamente realizada.

Por el contrario, las ciencias sí cuentan con "objeto externo", es decir, una perspectiva fuera de su quehacer que permite evaluarlo; esta disciplina cuenta con criterios de corrección que permiten verificar cuándo se realiza efectivamente ciencia y no otra cosa. En otros términos, la ciencia cuenta con la experiencia, a partir de la que puede verificar si se ha cumplido lo propuesto en una determinada hipótesis.

Por otra parte, la ciencia tiene, en cierta forma, de antemano aquello que pretende demostrar, esto es, el resultado ya se insinúa en la hipótesis. En cambio, como se vio, un pensar que se hace tal pensando no tiene nada de antemano. He aquí el escollo que no nos permite responder directamente a la pregunta "¿qué significa pensar?".

Al respecto, Heidegger afirma: “¿Cómo hoy puede afirmar alguien que nosotros no pensamos todavía, siendo que en todas partes hay un interés vivo por la filosofía, un interés cada vez más patente, siendo así que todos pretendemos saber qué pasa con la filosofía?" (GA 8, p. 6). Por lo tanto, lo importante que quiero destacar aquí es que más que referirse a cualquier pensamiento, Heidegger se refiere a la Filosofía; es en la filosofía donde ocurre el pensamiento en sentido propio, es decir, el pensamiento que estamos buscando. Al menos aquí, Heidegger determina un ámbito en el cual el pensamiento se desarrolla, la Filosofía.

A pesar de ello, por mucho que nos ocupemos con la filosofía, no es garantía de que realmente estamos pensando o filosofando. Lo que echa en falta Heidegger es un pensar en sentido propio, un pensar propiamente. Parecería que está hablando de cualquier modalidad del pensar, pero en realidad lo está haciendo respecto de un pensar que tiene su escenario en la filosofía.

En este sentido, Heidegger se pregunta si es el caso de que exista un pensar que no fuese propio, es decir, que fuese filosófico. Este autor afirma que sí, justamente en la ciencia. El filósofo afirma:

"En efecto, es cierto que lo dicho hasta ahora, y toda la exposición que ha de seguir, nada tiene que ver con la ciencia, y nada tiene que ver con ella precisamente si nuestra disquisición aspira a ser un pensar. El fundamento de este hecho está en que la ciencia por su parte no piensa, ni puede pensar, y por cierto, para su propio bien, o sea, para asegurar la propia marcha que ella se ha fijado. La ciencia no piensa. Esta afirmación resulta escandalosa, aun cuando apostillemos inmediatamente que, no obstante, la ciencia tiene que habérselas con el pensar en su propia forma especial. En cualquier caso, esa forma sólo es auténtica y en consecuencia fértil, si se hace visible el abismo que media entre el pensar y las ciencias, y que media entre polos infranqueables. Aquí no hay ningún puente, hay solamente un salto. Por eso son perjudiciales todos los puentes provisionales y los puentes de vía estrecha que 
precisamente hoy quieren instalar un cómodo tráfico recíproco entre pensar y las ciencias. Y, por tanto, nosotros ahora, en cuanto procedemos de las ciencias, hemos de soportar lo escandaloso y extraño del pensar, supuesto que estemos dispuestos al aprendizaje del mismo" (Ibíd, p. 19. Énfasis mío).

Aquí Heidegger expone su famosa afirmación que podría resultar provocadora e impropia y que motiva este artículo, a saber, "la ciencia no piensa". Sin embargo, lo cierto es que esta se ha malentendido como ninguna otra. Heidegger reconoce que la investigación científica pasa por su mejor y más productivo momento, en la ciencia sin duda hay pensamiento, lo que no significa que piense en sentido propio. Cuando Heidegger afirma que la ciencia no piensa, no se está pronunciando contra ella, sino que con ello intenta hacernos reflexionar en torno a la esencia de la ciencia. Por lo tanto, el pensamiento del que hace uso la ciencia, no es simplemente una condición deficiente del pensar en sentido genuino, sino que es un tipo de pensar que tiene su propia naturaleza, separada del pensar que le corresponde a la filosofía. Refiriéndose a esto Heidegger sostiene:

"Cuando se establece una diferencia, una distinción y separación entre el pensar y la ciencia -nos advierte-, eso se considera de inmediato como una degradación de la ciencia. E incluso se teme que el pensamiento inicie las hostilidades contra la ciencia y perturbe la seriedad y el agrado en el trabajo científico" (GA 8, p. 76).

Entonces, que la ciencia no piense se podría matizar a una frase como: "la ciencia no piensa en el sentido del pensar filosófico" (Acevedo, 2010, p.9). Para refrendar esta idea que delimita la ciencia y la filosofía en referencia a su modo de pensar, me gustaría recurrir a una entrevista realizada a Heidegger por Richard Wisser, en 1969, donde el filósofo afirma:

"Y esa frase, la ciencia no piensa, que causó gran sensación cuando la dije en una lección en Friburgo, significa: la ciencia no se mueve en la dimensión de la filosofía, pero está, sin que ella lo sepa, referida a esa dimensión. Por ejemplo, la física se mueve en espacio, tiempo y movimiento; lo que sea movimiento, lo que sea espacio, lo que sea tiempo, no lo puede decidir la ciencia en cuanto [als] ciencia; entonces, la ciencia no piensa; no puede en este sentido pensar con sus métodos. Yo no puedo, por ejemplo, con métodos físicos decir lo que sea la física. Lo que sea la física yo sólo lo puedo pensar a la manera del preguntar filosófico. La frase la ciencia no piensa no es ninguna objeción, sino solamente una constatación de la estructura interna de la ciencia; a su esencia pertenece que ella esté referida, por una parte, a lo que piensa la filosofía y, por otra parte, ella misma, sin embargo, olvida esto que hay que pensar y no lo considera" (1980, p. 178)

Aquí se establecen ciertos límites que la ciencia tiene por su propia naturaleza, la cual se funda sobre la base de ciertos conceptos y objetos, propios de una disciplina científica, 
esta disciplina piensa sus objetos y conceptos, se ocupa de ellos de una manera determinada, pero nunca pone en cuestión estos puntos de partida, sino que solo opera sobre ellos. La ciencia tiene, por una parte, un método definido que ha de fijar tanto el procedimiento de investigación como los criterios mediante los cuales la ciencia se da por satisfecha en sus resultados (la verificación en la experiencia); por otra parte, todos estos procedimientos operan sobre la base de un entramado de conceptos y objetos que fundan la investigación y sin la cual dicha investigación ni tendría objetos ni tendría método, pero que, por ello mismo, tampoco puede ponerlos en cuestión, ya que de lo contrario su tarea no tendría sentido. Definir lo que sea, por ejemplo, la física mediante la física misma no es posible, esta doctrina sólo realiza su quehacer en tanto da por supuesto sus métodos y objetos, si no, no habría nada. A raíz de esto, la reflexión en torno a los presupuestos de la ciencia solo es posible adoptando una actitud filosófica.

Por su parte, la filosofía no opera bajo los mismos supuesto, he ahí su dificultad y ambigüedad. Mas la filosofía sí puede pensar los fundamentos conceptuales de la ciencia. De manera que ocurre una situación un tanto curiosa, la disciplina que se encarga de pensar la ciencia no es la ciencia misma, sino la filosofía; por ello Heidegger afirma que la ciencia no piensa, no puede pensarse a sí misma, es decir, no puede pensarse desde sus fundamentos. No obstante, esto es así por el propio bien de la ciencia, dado que, de lo contrario no sería tal.

Lo importante a destacar aquí es que, en parte, con lo dicho hasta ahora se responde a las preguntas iniciales que nos planeamos como objetivo de este artículo, a saber:

1) Cualificar el sentido de pensamiento del que la ciencia no es partícipe: Aquí no se califica positivamente, sino de manera negativa, es decir, se intenta mostrar cuál es el pensar en sentido propio y con ello también mostrar que éste no es el pensar del que hace uso la ciencia para su quehacer. Hasta aquí es importante mencionar que no significa que la ciencia no piense en absoluto, sino que de cierta forma se relaciona con el pensar, es más, la naturaleza del pensar científico determina su forma de proceder, al igual que el pensar en sentido propio determina la forma de proceder de la filosofía.

2) El límite entre filosofía y ciencia: la diferencia entre estos dos ámbitos de pensar determina la distancia entre filosofía y ciencia, de manera tal que no es posible entenderlas en conjunto, es decir, ni una es reductible a la otra ni sus actividades son afines.

\section{CienCIA Y MEDITACIÓN}

Todavía queda pendiente delimitar ciencia y filosofía, en tanto tienen ámbitos distintos. Con este objetivo, creo que será relevante el breve texto de 
Heidegger "Ciencia y meditación" (2002). En él, Heidegger explica la naturaleza de la ciencia y, con ello, el sentido de la famosa afirmación "la ciencia no piensa".

El título de la obra puede oscurecer el asunto. Es importante aclarar en qué sentido debemos entender la conjunción " $\mathrm{y}$ " de "Ciencia y meditación", dado que, hasta ahora, hemos entendido que la ciencia no piensa propiamente, esto es, no “medita” sobre sus fundamentos. Como vimos en ¿Qué significa pensar?, realmente a ciencia y filosofía (meditación) los separa un abismo, es decir, son disciplinas que por su propia naturaleza se ocupan de asuntos distintos y con procedimientos radicalmente opuestos ${ }^{2}$. Heidegger presenta una especie de definición, sin serlo realmente, que sirve de punto de partida para iniciar la investigación en torno a la naturaleza de la ciencia moderna, el filósofo dice "la ciencia es la teoría de lo real" (GA 7, p. 43). De manera que la indagación de Heidegger se centra en el análisis etimológico de dos conceptos contenidos en esta frase, a saber, "real" (wirklich) y "teoría" (theorein).

Según este autor, "real" tiene su raíz en el término "wirken" (actuar, obrar). Esto, a su vez, lo conduce a afirmar que real "está entroncada también [con] la griega "thesis": posición, sitio, lugar" (GA 7, p. 44). ${ }^{3}$ Por lo tanto, Heidegger considera este obrar no solo como obrar humano, sino también como producto de la naturaleza, en tanto la naturaleza lleva a los objetos a nuestra presencia. De esta forma, el actuar del que se habla en un principio (wirken) debe entenderse como "presenciar". De manera que "realidad" quiere decir entonces, "lo que yace traído-ahí-delante, lo producido a la presencia". ${ }^{4}$

Es así como "lo real", que originariamente era pensado como lo plenamente presente a partir de un presenciar que reconocía y respetaba a lo que llevaba a la presencia, pasa a convertirse en "objeto". Como dicho objeto es objeto del representar

\footnotetext{
${ }^{2}$ Un punto de partida que nos podrá permitir delimitar la ciencia de la que habla Heidegger será recurrir a una importante cualificación que hace en este texto, es decir, de lo que se trata aquí es de la "ciencia moderna" y no de la ciencia en general. Por lo tanto, la physis griega o la doctrina medieval quedan fuera de esta consideración de "ciencia moderna". Evidentemente que, por ejemplo, la physis griega sigue teniendo relevancia en el presente, aunque tal parece que la forma de proceder de la ciencia moderna se aleja un tanto de la indagación griega, pero, por cierto, se funda en ella. Sin embargo, afirma Heidegger, todavía "permanecía oculto en la esencia del saber griegamente experimentado" (GA, 07, p. 43).

${ }^{3}$ Pido disculpas al lector por exponer la relación etimológica de las palabras analizadas por Heidegger de manera tan rápida, pero no quiero detenerme tanto en la consistencia de su procedimiento, sino que, más bien, en el resultado que de ahí obtiene el autor.

${ }^{4}$ También Heidegger recurre a otro camino para explicar el sentido originario de real, avanza de "Wirken" a "Werk" y a la griega ergon. El sentido originario de ergon-según el autor-se habría perdido y habría sido reemplazado por "obra" y "efecto", en palabras de Heidegger, "cuando los griegos, a saber, Aristóteles, hablaban de lo que los latinos nombraban causa efficiens, jamás se referían a realizar un efecto" (GA 7, p. 47). En cambio, el sentido propiamente aristotélico se relaciona con "lo que se produce en plena presencia" (ibid.).
} 
humano, resulta necesario reconocer una relación entre lo real y la teoría, entendida ésta como la expresión de dicho representar.

Nuevamente, tal como con el concepto de "real", Heidegger procede a hacer un análisis etimológico del concepto de "teoría" que rescate su sentido originario. Este concepto tiene su origen en el griego Theorein, entendido por Heidegger como "mirar el aspecto en el que lo presente aparece; demorarse en él viéndolo en tal visión" (GA 7, p. 49). El sentido griego de Theorein es el "auspiciar cuidadoso de la verdad" (GA 7, p. 49). Lo interesante es que, para Heidegger, mirar es des-cubrir en el pleno sentido de la palabra. De modo que, en la Theoria hay un esfuerzo por acceder a lo que se mantenía oculto al mero mirar desinteresado, que se preocupa por el advenimiento de lo presente y por el hecho de que dicho advenimiento mantenga intacta su verdad.

Sin embargo, según Heidegger, la teoría en un sentido moderno conserva poco de este sentido originario griego, lo cual se documenta en las traducciones del concepto de theoria al latín, que significa contemplación y en alemán Betrachtung (contemplación). De manera tal que mientras el sentido de teoría en griego que aspiraba a conservar lo real, ahora procede a una intervención y, con ello, acaba con el descubrimiento y con el llevado a la presencia. De forma que la teoría moderna "intenta agarrar, asir, asegurar lo real, adueñándose de ello" (GA 7, p. 52). En último término, el problema parece estar en que no es de algo otro de lo que intenta adueñarse, sino de algo que ella misma había elaborado (la teoría moderna).

Este es un punto que explica muy bien la naturaleza de la ciencia moderna y que la distingue de la filosofía. La ciencia moderna, en tanto teoría, reelabora lo real, de manera tal que "lo real es lo presente autoproducente" (GA, 07, p. 51) y lo presenta como objeto, que es resultado de una causa anterior, pero que, a su vez, es elaborado por el mismo hombre.

Con esto, Heidegger afirma algo interesante, la esencia de la ciencia moderna está en su método, es decir, solo mediante dicho método es posible la apropiación de lo real y dicho método consiste en medir y calcular. Lo que da pie para distinguir dos tipos de pensar diametralmente opuestos, el calculador y el meditativo. En este sentido, solo es posible el cálculo - que caracteriza a la ciencia moderna- cuando se cuenta con algo seguro y, en la medida que está seguro, ya se tiene de antemano, en un sentido moderno, como hipótesis.

Heidegger ilustra la naturaleza de la ciencia que extrajo del análisis de los conceptos de "real" y "teoría", con algunos ejemplos de ciencias particulares. Por ejemplo, afirma que el físico capta la naturaleza como inanimada y, con ello, solo aprehende un modo de aparecer de ella, de manera que es incapaz de captarla en su esencia. En la física atómica -explica Heidegger- "la teoría sigue estando consignada a que el átomo se ex-ponga en una percepción sensible" (ibid. p. 169); 
la teoría nunca puede abarcar la naturaleza en su plenitud. El hombre solo construye cercos en lo que le interesa, pero con ello la naturaleza como tal resulta inabarcable. Por eso la ciencia de la naturaleza está sometida a exponer siempre solo un modo como presente y nunca la naturaleza tal como es en su esencia. Según Heidegger la naturaleza es inabarcable en un doble sentido:

1. Inabarcable por cuanto la teoría siempre está sometida a lo presente.

2. Tampoco es abarcable por cuanto la aspiración a la objetividad, es decir, buscar lo real como una cosa, impide que su correspondiente "concebir" y "asegurar" nunca pueda disponer de la plenitud esencial de la naturaleza.

La idea del autor es que la ciencia nunca podrá decidir si la naturaleza se retrae o se muestra esencialmente, ni siquiera le corresponde plantearse dicho problema -afirma Heidegger-, ya que la teoría siempre estará limitada al ámbito de la objetividad. Por lo tanto, la naturaleza calculadora de la ciencia moderna no puede pensar su esencia (asunto fundamental que permite distinguir esta ocupación de la filosófica), sino que está limitada por sus métodos. Esta limitación impide a la ciencia realizar un discurso sobre sí misma, así lo explica Acevedo:

"Así como las ciencias no pueden autodefinirse mediante sus métodos -eso es lo que hemos querido establecer o sugerir-, tampoco pueden a través de ellos definir suficientemente aquello sobre lo que versan. La naturaleza, en el caso de la física; la historia, en el caso de la historiografía; el hombre, en el de la psiquiatría; la lengua, en el de la filología. Todo eso -naturaleza, historia, hombre, lengua-, pertenece a lo que para las ciencias queda como inabarcable o irrodeable" $(2010$, p. 12).

La ciencia, en tanto proceder calculador, siempre limita las posibilidades de los objetos, limitándolo y ocupándolos para fines específicos. Por ejemplo, si ocupamos un rio para producir energía hidroeléctrica, se estará considerando sólo en tanto puede producir dicha energía, pero absurdo pensar que en ello consiste su esencia. Por eso, dicho proceder, siempre se le escapa algo que queda como inabarcable.

La meditación que ha llevado a cabo Heidegger sobre la ciencia ha consistido principalmente en una búsqueda del sentido de ella. Justamente el sentido (Sinn) es lo esencial de la meditación (Besinnung). Pero es claro que la búsqueda del sentido no consistirá jamás en la búsqueda de algo asible, de suerte que una vez llegados al final del camino pudiéramos agarrarlo, éstas son pretensiones propias de la ciencia moderna, no de la meditación. Mientras que el pensar calculador propone algo desde antemano con el objetivo de asegurarlo en su obtención, la meditación (filosofía o pensar) no propone nada, deja ser, es decir, quién medita no propone nada sobre lo cual tematizar, sino que deja que lo esencial se muestre por sí mismo y se exponga en su propia naturaleza (cf. GA, 07, p. 65).

Con lo que podemos afirmar que la meditación se opone a cualquier objetivo determinado, es decir, no tiene la forma del proceder científico, 
el cual orienta su quehacer en vistas de un objetivo o la producción de algo. Una meditación que adoptara esta naturaleza técnica no tendría como objetivo dilucidar su propia esencia, sino que sus esfuerzos estarían centrados en otro que ella misma, esto es, en su resultado.

\section{Filosofía COMO AUtOCONCIENCIA}

Hasta aquí hemos distinguido la filosofía de la ciencia, pero, si bien hemos dado algunos atisbos, todavía no queda muy claro cuál es la relación entre la filosofía y la autoconsciencia. A pesar de que el desarrollo de esta idea sería, por sí sola, tema de una investigación más extensa, me gustaría dejar planteadas ciertas ideas con el fin, no tanto de realizar una argumentación en torno a la autoconciencia misma, sino con el objetivo de aclarar el particular carácter reflexivo del cual carece la ciencia y que caracteriza al pensar y la filosofía.

No hay que perder de vista que el tema del autoconocimiento ${ }^{5}$ prácticamente da inicio a la filosofía a partir del relato de oráculo délfico en la Apología, donde se declara que Sócrates es "sabio en sumo grado" (21b5). No obstante, esta sabiduría no consiste en saber algo determinado o delimitado por un cierto objeto, sino que "la certeza de no creer saber lo que no sabe es precisamente saber en grado sumo y que, por consecuencia, es posible la descalificación de otras actividades que solo pretenden ser un saber" (Abalo, 2015, p. 12; cf. 21d). En este caso, "saber" es "ser consciente de ignorar algo" (ibid.) y es diferente a la manera habitual de no saber, puesto que generalmente decimos que alguien no sabe algo cuando lo ignora absolutamente, es decir, ignora los límites de su propio conocimiento. El peligro de esta ignorancia, según Sócrates, es que quien no sabe de esta forma, puede sobreestimar su propio conocimiento y a partir de esto errar. De forma tal, que la solución a este problema justamente es ser consciente de nuestros propios límites o bien, si se quiere, tener un conocimiento sobre el conocimiento en sentido general.

Otro pensador que detectó esto de una manera más precisa fue Kant, quien afirmó en la Crítica de la razón pura:

"Es manifiesto que no es efecto de la ligereza, sino de la madura facultad de juzgar propia de la época, [época] que no se deja entretener más con un saber ilusorio; y es una exigencia planteada a la razón, de que ésta vuelva a emprender la más fatigosa

\footnotetext{
${ }^{5}$ En adelante intentaré a la larga cambiar el término autoconocimiento por autoconsciencia tratando de considerar el estatuto general de dicha indagación, esto es, no se trata de un conocimiento determinado sobre uno mismo en cuanto objeto u otro objeto distinto de uno; sino que de una forma de consciencia de lo que somos en tanto una determinada entidad (cf. Placencia, 2015, p. 546). Además, me parece que autoconsciencia, da cuenta mejor de que no se trata de una indagación externa a uno mismo, sino que se trata más bien de una investigación en torno a lo que la tradición ha llamado "uno mismo" (cf. Smith, 2020).
} 
de todas sus tareas, a saber, el conocimiento de sí; y de que instituya un tribunal de justicia que la asegure en sus pretensiones legítimas, y que por el contrario pueda despachar todas las $\mid$ arrogaciones infundadas, no mediante actos de autoridad, sino según sus leyes eternas e invariables" ( $\mathrm{KrV}$, A xi-xii).

Este tribunal de justicia tampoco obtendrá un conocimiento particular de algún objeto, sino que "la determinación, tanto de sus fuentes, como del alcance y de los límites" (KrV, Axii) de este conocimiento de segundo orden, que este autor llama "trascendental" (KrV, B25). Placencia (2015, pp. 548-9) explica la función de este autoconocimiento de Kant de manera no muy distinta a como parecía verla Sócrates, a saber:

“[En Kant] el autoconocimiento sería algo así como una tarea necesaria para evitar un tipo de error filosófico, $s c$. el error metafísico en el que la razón humana incurre, según Kant, de modo natural y filosóficamente motivado. Tal error, que como se sabe, (...) estaría generado justamente por una falta de conocimiento respecto de los límites de nuestras capacidades cognitiva. (...) la tarea de autoconocimiento que arriba denominé típica o general, pareciera ser su objeto en un sentido aún más amplio. De hecho, lo es, tal como cabe notar a partir de las Lecciones de lógica, de acuerdo al texto editado por su discípulo G. B. Jäsche, Kant hace notar que la pregunta por el tipo de ser que somos, $s c$. la pregunta por el hombre constituye algo así como el crisol en que se funden las preguntas centrales de la filosofía en sentido cosmopolita (AA 09 25, 1-6)" ${ }^{6}$

Con lo cual, la pregunta no solo tiene que ver con la filosofía, sino que con ella en cuanto esta última tiene una íntima relación con nosotros mismos.

\section{Continuidad en Heidegger del proyecto de la filosofía COMO AUTOCONCIENCIA}

Esto no se distancia tanto de lo propuesto por Heidegger en los textos revisados, ya que la afirmación "la ciencia no piensa" nos proporciona pistas para entender, no solo el sentido concreto de la afirmación específica, sino que ayuda a comprender en qué consiste la actividad filosófica (y por lo tanto, la del propio Heidegger, en tanto filósofo) así como la actividad científica. Hemos visto que sobre la base de la afirmación "la ciencia no piensa" está supuesto el reconocimiento y distanciamiento de dos ámbitos distintos, el del pensamiento calculador (científico) y el del meditativo (filosófico). Dichos pensamientos se distinguen, como hemos visto, debido a su objeto (uno determinado y otro indeterminado), de sus criterios de corrección (empírico o externos e internos o filosóficos).

\footnotetext{
${ }^{6}$ Para profundizar en esto, cf. Tugendhat (1979).
} 
Por una parte, el quehacer filosófico se sustenta en la correspondencia con algo otro, a saber, el ser. Hemos visto como dicha correspondencia tiene una relación bilateral, en la que no solo se espera que el ser humano activamente encuentre los fundamentos de lo real, sino que esa búsqueda depende de que "lo real" se aparezca de una determinada manera por sí misma. En esta medida aquello a lo cual se refiere la filosofía es externo a la actividad misma, es decir, la esencia que espera alcanzar sobrepasa la facticidad de un determinado objeto u hombre.

Mientras que el quehacer científico no refiere a algo distinto de su propia actividad y está limitado por ella misma, de manera tal que sus resultados se esperan y se cuentan de antemano al inicio de la actividad misma de la ciencia. La ciencia, afirma Heidegger, se ocupa de un modo particular del ser y además permanece ciega a cualquier indagación que rebase dicha particularidad. Por lo tanto, la actividad científica misma, vista desde su interior, se somete a un método determinado y definido que no hace sino producir un movimiento circular en el cual se propone una hipótesis -bajo los límites de la forma objetual (Gegenstand) - y se obtiene justamente aquello que se había propuesto en un inicio y bajo la forma que se esperada y no otra.

Por este motivo, a pesar de que la referencia, en la filosofía parece ser externa y en la ciencia parece ser interna, no ocurre lo mismo con los criterios de corrección. Es decir, los criterios de corrección de la filosofía son internos y por este motivo la denomino reflexiva o autoconsciente; mientras que los de la ciencia son externos. En torno a lo primero, funciona el paralelo con Kant y su Tribunal de la razón (KrV, Axi-xii), puesto que se aprecia que sendos autores concebían que la filosofía no podía obtener sus criterios de corrección sino de ella misma, en tanto se trata de una disciplina que versa, en algún sentido, sobre sí misma, sus límites y posibilidades y no sobre objetos directamente -aunque, por supuesto, pueda referir legítimamente a ellos con posterioridad.

Para que el quehacer filosófico dé cuenta de sí mismo, no puede recurrir, como la ciencia, a elementos externos al planteamiento de la hipótesis, a saber, a la experiencia. Sin embargo, puede regirse por las propias normas o caminos que le plantea su propia actividad con el objetivo de descubrirse a sí misma. En Heidegger se conserva este espíritu de auto-comprensión kantiano-socrático, por ejemplo, en su texto "¿Qué es metafísica?" comienza indicando que el título del mismo parece sugerir que "se hablará sobre metafísica", pero, en vez de ello, "discutiremos una pregunta metafísica" (GA 09, p. 103). Por lo que al igual que en ¿Qué significa pensar?, el modo de proceder con su objeto se sitúa in media res, esto es, no se intenta responder la pregunta directamente como si se intentase dar una definición de lo que es pensar, sino que, en vez de eso, Heidegger habla sobre el pensamiento intentando mostrar una guía (una senda en el bosque) para responder la pregunta, sin 
hacerlo directamente, ya que, dar una respuesta, en cierta medida sería someterse a la naturaleza de la actividad científica.

En cambio, en cierta medida, la ciencia no descubre nada distinto de sí misma, en tanto la hipótesis determina de antemano la forma de su resultado, en esta medida cualquier verificación tendrá forma objetual. Sin embargo, no sucede lo mismo con los criterios de corrección de la verificación, esto es, la piedra de toque con la que se constata la hipótesis se obtiene bajo el examen de algo otro que la hipótesis misma, a saber, la experiencia. En este caso, la experiencia es algo con lo que se enfrenta la hipótesis y que, hasta cierto punto, no se puede anticipar.

Este paso es complicado, puesto que lo que es tomado por externo aquí, puede ser tomado por interno en otro sentido, como vimos antes. Externo e interno aquí refiere a la naturaleza de los criterios de corrección. Por una parte, tenemos que la ciencia inicia su actividad con la postulación de una forma de ser de la realidad, la cual ella misma no percibe directamente, sino que anticipa o postula. Por otra parte, el resultado de lo que se anticipa se tiene de antemano (de ahí que llamaba a la referencia interna), pero además, lo que se aparece en la realidad no es el postulado mismo, el cual, en cierta medida depende de la actividad individual de un agente que la formula de esa manera, sino que proviene de un fenómeno constatable y que es posible exponer ante otros, de manera tal que otros agentes pueden constatar también que aquello sea el caso o no -obviamente limitado por la forma objetual-.

Esta forma de objetividad se fundamenta en la naturaleza del criterio de corrección, a saber, la experiencia. Si la experiencia no fuera, en algún sentido, distinta de la hipótesis (cuya naturaleza es más bien conceptual que real), entonces no podría ser considerada por otros de esta manera (como hipótesis), sino que solamente sería tenida para sí misma como verdadera, ${ }^{7}$ es decir, no sería un hecho reconocible por otros.

Justamente la forma limitada y establecida de los criterios de corrección de la ciencia da lugar a su éxito y a que el quehacer científico ocupe un alto sitial en nuestra época. No obstante, el problema aparece cuando, la ciencia no busca realmente "descubrir" en sentido heideggeriano, sino es susceptible de caer en error en la medida en que no es capaz de dar cuenta de los límites de dicho quehacer y, por este motivo, sobreestima su aproximación al objeto, tal como los individuos interrogados por Sócrates en su Apología.

En último término, el pensar, en sentido heideggeriano, debe cumplir una función reflexiva o autoconsciente y no meramente productiva, es decir, no sólo se

\footnotetext{
${ }^{7}$ Esto no significa que una forma de verdad que sea válida solo para un solo individuo sea, por ello, una forma de verdad menos legítima, al contrario, podría ser, en términos de Heidegger una forma de experiencia que capte mejor el ser que el proceder científico. Es decir, una perspectiva como ésta podría captar mejor el proceder científico que la propia ciencia (la filosofía)
} 
debe orientar a ocuparse de un objeto específico y obtener resultados con él, sino que también debe indagar en su propia actividad de aproximarse a ese objeto $\mathrm{y}$, en cierta medida, de su correspondencia con el mismo, es decir, su propia esencia.

Esta reflexión da cuenta de un discurso no tanto sobre el objeto mismo, sino sobre el conocimiento mismo que analiza los criterios de corrección del conocimiento y, a través de esto, de la correspondencia de esos criterios con la naturaleza humana.

En este sentido, 1) la pregunta por el conocimiento de un objeto, pasa a 2) la pregunta por el conocimiento del conocimiento, lo que estos autores han llamado filosofía y, finalmente, 3) a la pregunta por la correspondencia de es a filosofía con nosotros mismos, es decir, entender a la filosofía como una forma de autoconocimiento, es decir, una actividad mediante la cual no solo nos ocupamos de objetos, sino en la que nos hacemos objetos a nosotros mismos mediante esa investigación.

\section{BIBLIOGRAFÍA}

Abalo C, Francisco. (2015): "El problema de determinar la "sabiduría" en los primeros diálogos de Platón". Revista de filosofía, 71, pp. 9-21.

Acevedo, Jorge (2010) "La frase de Heidegger "la ciencia no piensa", en el contexto de su meditación sobre la era técnica”. Revista de Filosofía, 66, pp. 5 -23.

Kant, Immanuel (2010) Crítica de la razón pura. Madrid: Fondo de cultura económica.

Heidegger, Martin (2015) Ser y tiempo. Santiago: Ed. Universitaria.

Heidegger, Martin (2010) ¿Qué significa pensar?, Madrid: Ed. Trotta.

Heidegger, Martin (2002): Serenidad, Barcelona: Ediciones del Serbal.

Heidegger, Martin (1997): “Ciencia y meditación”. En Filosofía, Ciencia y Técnica, Ed. Universitaria, Santiago.

Heidegger, Martin (1990): “La constitución onto-teo-lógica de la metafísica". En Identidad $y$ diferencia, Barcelona: Anthropos.

Heidegger, Martin (1980): "Martin Heidegger en diálogo". En: García de la Huerta, L. M. La Técnica y el Estado Moderno. Santiago: Departamento de Estudios Humanísticos de la Universidad de Chile. 
Heidegger, Martin (1974 y ss.): Gesammtausgabe (=GA). Frankfurt am Main: Klostermann.

Placencia, L. (2015) “Autoconocimiento, acción y autointerpretación. Opacidad y transparencia del saber de sí mismo en la filosofía práctica de Kant”, Anuario Filosófico, 48,3 , pp. 543-563.

Platón (2002): Apología de Sócrates. Santiago de Chile: Editorial Universitaria.

Smith, Joel (2020): "Self-Consciousness", The Stanford Encyclopedia of Philosophy (Summer 2020 Edition), Edward N. Zalta (ed.), URL = <https://plato.stanford.edu/archives/ sum2020/entries/self-consciousness/>.

Tugendhat, E. (1979): Selbstbewußtsein und Selbstbestimmung. Frankfurt am Main: Suhrkamp. 